\title{
A Continuous Monitoring System for Total Mercury in Waste Water by Cold Vapor Atomic Absorption Spectrometry and Continuous Microflow Analysis
}

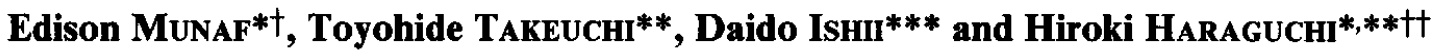 \\ * Department of Applied Chemistry, School of Engineering, Nagoya University, Nagoya 464-01, Japan \\ **Research Center for Resource and Energy Conservation, Nagoya University, Nagoya 464-01, Japan \\ *** Department of Industrial Chemistry, Kumamoto Institute of Technology, \\ Ikeda, Kumamoto 860, Japan
}

\begin{abstract}
An automated system based on "continuous microflow analysis" and cold vapor atomic absorption spectrometry has been developed for monitoring the concentration of total mercury at sub- $\mu \mathrm{g} / 1$ levels. The method involves a continuous microflow technique for sample digestion, reduction and extraction in small bore tubes $(0.5 \mathrm{~mm}$ i.d.) at $\mu \mathrm{l} / \mathrm{min}$ flow rates of reagents. Sodium tetrahydroborate in sodium hydroxide solution was used as the reducing reagent. In the case of discrete injection of sample $(200 \mu \mathrm{l})$, analysis time for the determination of total mercury was about 3 min per sample. The detection limit of mercury(II) was $0.15 \mu \mathrm{g} / \mathrm{l}(S / N=3)$ for a gas flow cell of $40 \mathrm{~mm}$ path length. The relative standard deviation was $0.99 \%$ at $4.0 \mu \mathrm{gHg} / 1(n=10)$. The calibration curve was linear up to $5.0 \mu \mathrm{g} / 1$. Under the optimal conditions, the present method could be successfully applied to the continuous monitoring of total mercury in waste water.
\end{abstract}

Keywords Microflow analysis, cold vapor atomic absorption spectrometry, mercury determination, waste water, continuous monitoring

Interest in the determination of mercury stems principally from its toxicity to humans in industrial and laboratory exposure. Therefore, the determination and monitoring of total mercury in environmental samples are extremely important. The cold vapor atomic absorption spectrometric technique, based upon the reduction of mercury by tin(II) chloride is the most common method for determining mercury in environmental samples. Since its introduction by Hatch and $\mathrm{Ott}^{1}$, the reliability and ease of operation of the method have been considerably improved.

The unsegmented flow techniques offer a wide range of possible methodologies. Thus conventional flow injection analysis (nFIA, injection of sample into a continuous flow of reagent) allows analysis of pollutants with minimum consumption of the sample solution; here the reagents demand the major expenditures for determination. In cases such as waste water analysis, reversed FIA (rFIA) is thus more suitable to reduce the analytical cost. On the other hand, when continuous monitoring of the varied analyte concentrations in samples is required, the completely continuous flow method (continuous recording of the signal produced) is

$\dagger$ On leave from Department of Chemistry, Faculty of Mathematics and Natural Sciences, Andalas University, Padang, West Sumatra, Indonesia.

\#To whom correspondence should be addressed. advantageous.

We have reported use of a microflow technique for the completely continuous determination of total mercury as well as the speciation of mercury compounds. ${ }^{2-6} \quad$ Tin(II) chloride in alkaline medium was used as a reducing reagent in these methods.

Sodium tetrahydroborate can be used as a reduction reagent for a wide variety of metal ions ${ }^{7-10}$, including mercury. The present paper reports a thorough study on the use of sodium tetrahydroborate as a reducing agent for determining mercury by continuous microflow analysis with cold vapor atomic absorption spectrometric detection.

\section{Experimental}

\section{Apparatus}

All glassware used in the present work was made of Pyrex glass. The pieces were first cleaned with aqua regia, and then rinsed and filled with $0.1 \mathrm{~mol} / \mathrm{l}$ hydrochloric acid for $1 \mathrm{~d}$. The cleaned glassware pieces were then washed twice with ultrapure water. Standard mercury solutions were prepared in volumetric flasks with glass stoppers and stored in a refrigerator.

The schematic diagram of the continuous microflow monitoring system for the determination of mercury is shown in Fig. 1. The instrumental components and 
Table 1 Instrumental components and operating conditions

\begin{tabular}{ll}
\hline Peristaltic pumps & Minipuls 2, Gilson, France \\
Sample & $200 \mu \mathrm{l} / \mathrm{min}$ \\
Oxidizing reagent & $50 \mu \mathrm{l} / \mathrm{min}$ \\
Catalytic reagent & $50 \mu \mathrm{l} / \mathrm{min}$ \\
Reducing reagent & $100 \mu \mathrm{l} / \mathrm{min}$ \\
Spectrophotometer & UVD-2, Shimadzu, Japan \\
Flow cell & $40 \mathrm{~mm}$ (path length), 6 mm \\
& i.d., with quartz windows \\
Mercury lamp & LC-2, Shimadzu, Japan \\
Chart recorder & R-20, Rika Denki, Japan \\
Gas-liquid separator & laboratory-made \\
Refrigerated circulating bath & RTE-5b, Neslab, Japan \\
Flow meter & UB-13, Settsu Kinzoku, \\
& Japan \\
\hline
\end{tabular}

operating conditions used are listed in Table 1.

\section{Chemicals}

All reagents were of analytical reagent grade or better grade. They were obtained from Wako Pure Chemicals Co. (Japan), unless otherwise noted. The water used was ultrapure water (prepared by Millipore, Milli Qwater purification system). The reagents and their concentrations were as follows:

Standard mercury solutions. The mercury standard solutions in the concentration range $0.5-4.0 \mu \mathrm{g} / 1$ were prepared from commercially available stock solution in $0.1 \mathrm{~mol} / 1$ hydrochloric acid.

Potassium peroxodisulfate solution $(2 \%(w / v))$. Prepared by dissolving $2 \mathrm{~g}$ of potassium peroxodisulfate in $100 \mathrm{ml}$ of water.

Cadmium chloride solution (100 $\mathrm{mgCd} / \mathrm{l}$ ). Prepared by dilution of $1.000 \mathrm{mgCd} / 1$ stock solution with water.

Sodium tetrahydroborate solution (2\% in 2 mol/l sodium hydroxide solution). Prepared by dissolving $2 \mathrm{~g}$ of sodium tetrahydroborate powder (Katayama Chemical Co., Japan) in $2 \mathrm{~mol} / 1$ of sodium hydroxide solution.

Mercury vapor adsorbent. Mercury vapor adsorbent (Shibata Kagaku Co., Japan) was used to prevent atmospheric contamination.

\section{Recommended procedure}

The standard procedure for the determination of total mercury is as follows. The sample, potassium peroxodisulfate reagent, cadmium reagent and sodium tetrahydroborate reagent are continuously injected via peristaltic pumps at the flow rates of 200, 50, 50 and $100 \mu \mathrm{l} / \mathrm{min}$, respectively. The sample stream is first mixed with the potassium peroxodisulfate reagent, then with the cadmium reagent, and then introduced into an oxidation reaction PTFE tube $(0.5 \mathrm{~mm}$ i.d., $27 \mathrm{~cm}$ long), where the organic mercury is converted to $\mathrm{Hg}$ (II) ions. The oxidation reaction in this step is performed at room temperature, and thus no heating digestion bath is used. ${ }^{2}$ The stream is then mixed with the reducing reagent and led into a reduction reaction PTFE tube $(0.5 \mathrm{~mm}$ i.d.,
$4 \mathrm{~cm}$ long), where the reduction reaction takes place. The generated mercury vapor is swept by the argon gas at a flow rate of $9.0 \mathrm{ml} / \mathrm{min}$, and introduced into a reduction and extraction PTFE tube $(1.3 \mathrm{~mm}$ i.d., $60 \mathrm{~cm}$ long). At this stage the $\mathrm{Hg}(\mathrm{II})$ ion is reduced to atomic mercury and extracted into the gas phase. The mixture is then introduced into the laboratory-made gas-liquid separator, as described in the previous paper ${ }^{11}$, and the mercury vapor dried through a condenser is then passed into the photometric flow cell $(40 \mathrm{~mm}$ path length and $1.13 \mathrm{ml}$-volume). The atomic absorption of mercury in absorbance at $253.7 \mathrm{~nm}$ is continuously recorded to monitor the total amount of mercury in sample solutions.

\section{Results and Discussion}

\section{Optimization of reagent flow rates}

The flow rates of the reagents in the mercury atomic absorption detection were optimized by using a solution of $2 \%$ potassium peroxodisulfate, a cadmium solution of $100 \mathrm{mgCd} / 1$, and a solution of $2 \%$ sodium tetrahydroborate in $2 \mathrm{~mol} / 1$ sodium hydroxide solution. These solutions were injected into the sample solution at valves 1, 2 and 3 in Fig. 1.

When the flow rate of the reducing reagent, i.e., $2 \%$ sodium tetrahydroborate in $2 \mathrm{~mol} / 1$ sodium hydroxide solution, was changed from 25 to $250 \mu \mathrm{l} / \mathrm{min}$, almost constant response was observed at the flow larger than $100 \mu \mathrm{l} / \mathrm{min}$ (Fig. 2A). Therefore, $100 \mu \mathrm{l} / \mathrm{min}$ was chosen as the flow rate of the reducing reagent. In this experiment the flow rates of potassium peroxodisulfate, cadmium chloride and the sample (mercury(II) chloride) solutions were kept constant at 50,50 and $200 \mathrm{l} / \mathrm{min}$, respectively.

Figure 2(B) shows the effect of the flow rate of the sample solution on the signal response. In this case, the flow rates of argon gas, sodium tetrahydroborate, potassium peroxodisulfate and cadmium chloride were kept

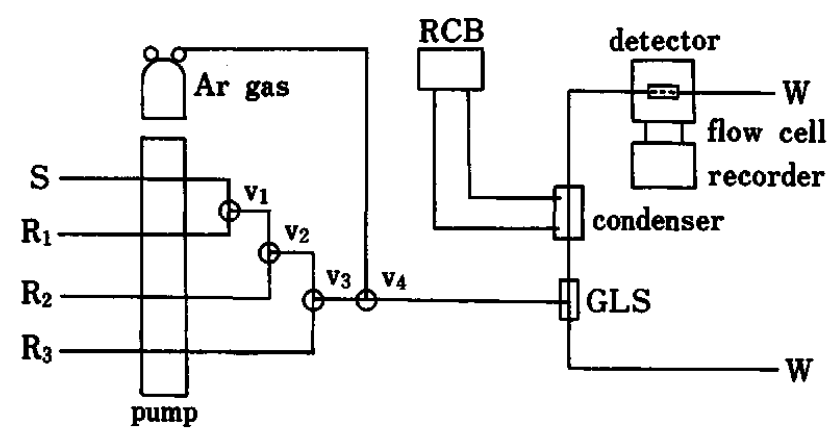

Fig. 1 Schematic diagram of continuous flow monitoring apparatus for total mercury. $R_{1}$, oxidizing reagent; $R_{2}$, catalytic reagent; $R_{3}$, reducing reagent; $S$, sample; $v_{1}, v_{2}, v_{3}$, $v_{4}$ : valves; GLS, gas-liquid separator; $R C B$, refrigerated circulating bath; $\mathrm{W}$, waste. 

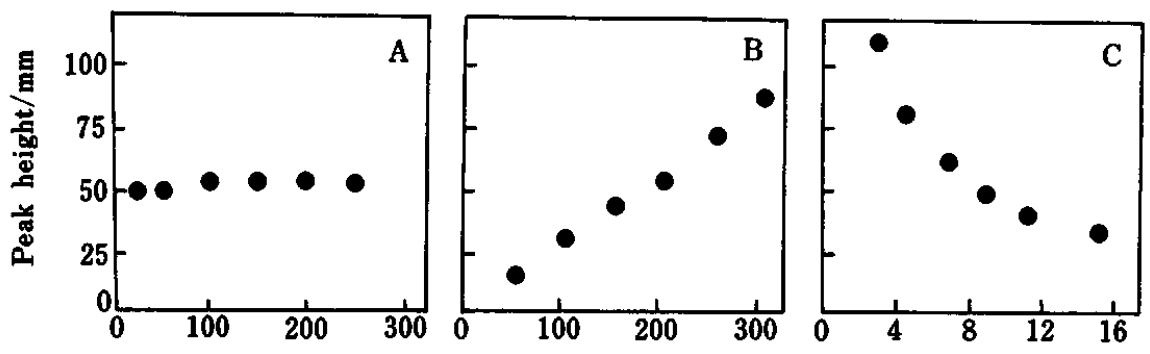

Flow rate $/ \mu \mathrm{l} \min ^{-1}$

Fig. 2 Dependences of analytical sensitivities on flow rates of reducing reagent (A), sample solution (B) and argon gas (C). (A) Sample solution, $200 \mu \mathrm{l} / \mathrm{min}$; argon gas, $9.0 \mathrm{ml} / \mathrm{min}$. (B) Reducing reagent, $100 \mu \mathrm{l} / \mathrm{min}$; argon gas, $9.0 \mathrm{ml} / \mathrm{min}$. (C) Sample solution, $200 \mu \mathrm{l} / \mathrm{min}$; reducing reagent, $100 \mu \mathrm{l} / \mathrm{min}$.

constant at $9 \mathrm{ml} / \mathrm{min}, 100,50$ and $50 \mu 1 / \mathrm{min}$, respectively. When the flow rate of the sample solution was changed from 50 to $300 \mu 1 / \mathrm{min}$, the signal response increases with increasing the flow rates. However, at flow rates larger than $200 \mu 1 / \mathrm{min}$, the gas flow of argon was not stable due to a large pressure drop across the $\mathrm{T}$ joint $\left(\mathrm{V}_{4}\right)$ in Fig. 1 . For this reason $200 \mu \mathrm{l} / \mathrm{min}$ was selected as the sample flow rate.

Figure 2(C) shows the relationship between the argon gas flow rate and the absorption response of mercury. When the flow rate of argon gas was changed from 3 to $15 \mathrm{ml} / \mathrm{min}$, the absorbance signal decreased with increasing the argon gas flow rate. However, the signal was not stable at any flow rate of argon lower than $9 \mathrm{ml} /$ $\min$. Therefore, $9 \mathrm{ml} / \mathrm{min}$ was chosen as the optimal flow rate. In this experiment the flow rate of sample, potassium peroxodisulfate, sodium tetrahydroborate and cadmium chloride were kept constant at the levels of $200,100,50$ and $50 \mu \mathrm{l} / \mathrm{min}$, respectively.

\section{Optimization of reagent concentrations}

Since Fernandez ${ }^{12}$ introduced sodium tetrahydroborate as a reductant for mercury vapor generation in 1973, use of sodium tetrahydroborate for vapor generation technique has become a popular method in ultratrace analysis of mercury. ${ }^{13-17}$ In the present work, the effect of the concentration of sodium tetrahydroborate on the signal response in microflow analysis was investigated. The concentration of sodium hydroxide was kept constant at $2 \mathrm{~mol} / \mathrm{l}$. A $4 \mu \mathrm{g} / \mathrm{l}$ mercury standard solution (mercury(II) chloride) and ultrapure water were alternately injected for 10 min. As shown in Fig. 3(A), when the concentration of sodium tetrahydroborate was changed from 0.5 to $5.0 \%$, the sensitivity of mercury increased up to the $2 \%$ and remained constant at the higher concentration. Consequently, $2 \%$ was selected as the optimum concentration.

Figure 3(B) shows the effect of the concentration of sodium hydroxide on the signal response of mercury. When the concentration was changed from 0.5 to $5.0 \mathrm{~mol} / \mathrm{l}$, the mercury signal increased with increasing
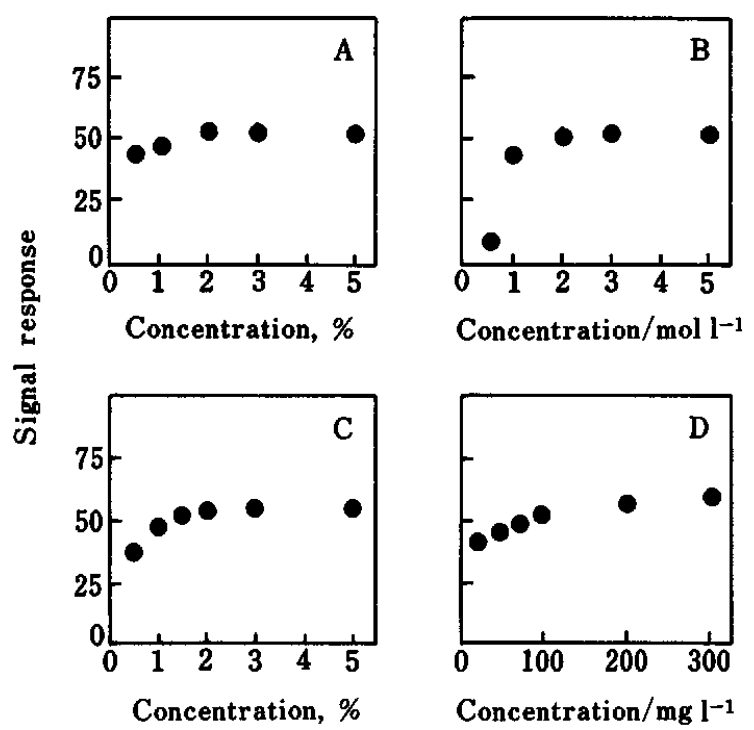

Fig. 3 Dependences of analytical sensitivities on concentrations of sodium tetrahydroborate, sodium hydroxide, potassium peroxodisulfate, and cadmium chloride. (A) Concentration of sodium tetrahydroborate in reducing reagent was varied. (B) Concentration of sodium hydroxide in reducing reagent was varied. (C) Concentration of potassium peroxodisulfate was varied. (D) Concentration of cadmium chloride was varied. Reagent concentrations (except for the reagents described in (A), (B), (C) and (D)): $2 \%$ sodium tetrahydroborate, $2 \mathrm{~mol} / 1$ sodium hydroxide, $2 \%$ potassium peroxodisulfate, $100 \mathrm{mg} / 1$ cadmium chloride.

the concentration, and reached a constant value at concentrations higher than $2 \mathrm{~mol} / \mathrm{l}$. Therefore, $2 \mathrm{~mol} / 1$ was chosen as the sodium hydroxide concentration.

Oda and Ingle ${ }^{18}$ reported that the analytical sensitivity of mercury, analyzed by cold vapor atomic absorption spectrometry, increased when the sample solution was oxidized prior to reduction with sodium tetrahydroborate. We observed a similar phenomenon by using potassium peroxodisulfate as the oxidant and tin(II) 
chloride as the reductant. ${ }^{2}$ In the present experiment, we have thus investigated the effect of potassium peroxodisulfate on the atomic absorption of mercury by using sodium tetrahydroborate as a reducing reagent. As can be seen in Fig. 3(C), when the concentration of potassium peroxodisulfate was changed from 0.5 to $5 \%$, the sensitivity of mercury increased in the concentration range of 0.5 to $2 \%$ and remained constant at concentrations higher than $2 \%$. Therefore, $2 \%$ potassium peroxodisulfate was chosen as the optimum concentration.

The reduction of mercury in alkaline medium is accelerated by addition of copper(II) ${ }^{2-6,16}$, or cadmium(II) salts. ${ }^{19}$ Thus, the effect of cadmium(II) on mercury generation was examined in concentrations from 50 to $300 \mathrm{mg} / \mathrm{l}$ in this work. The result is shown in Fig. 3(D). The mercury signal response increased with increasing the cadmium concentration and reached a constant value at concentrations higher than $100 \mathrm{mg} / \mathrm{l}$. Therefore, the optimum concentration of cadmium was set at $100 \mathrm{mg} / \mathrm{l}$.

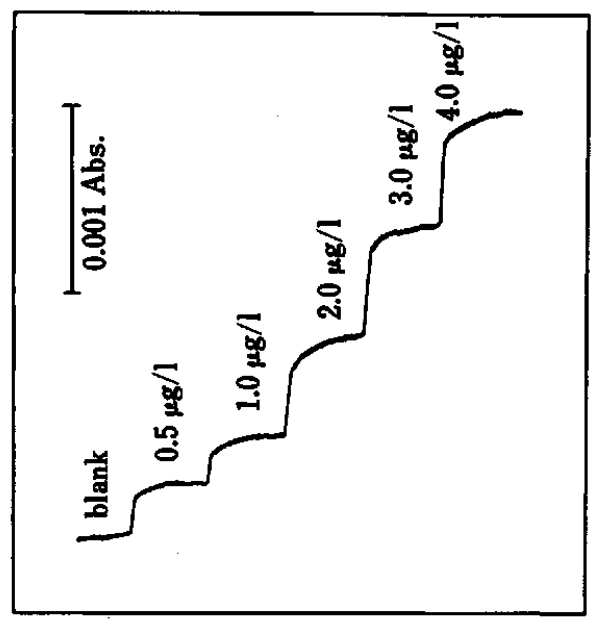

Fig. 4 Calibration run for the determination of mercury by completely continuous monitoring. Concentration of sample, $4.0 \mu \mathrm{gHg} / \mathrm{l}$; reagents used: $2 \%$ potassium peroxodisulfate, $100 \mathrm{mgCd} / 1,2 \%$ sodium tetrahydroborate in $2 \mathrm{~mol} / 1$ sodium hydroxide solution. Other conditions as in Table 1.

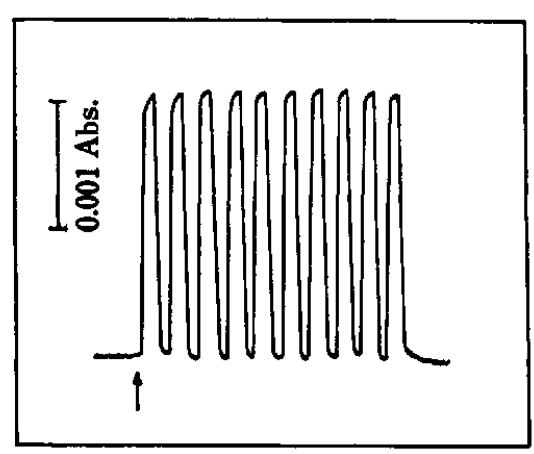

Fig. 5 Replicate injections of $4.0 \mu \mathrm{g} / 1$ inorganic mercury. Conditions as in Fig. 4.

\section{Analytical figure of metal}

Using the optimal conditions described above, the analytical figures of merit in mercury determination by the present method were evaluated. Figure 4 shows the calibration run for mercury. In the concentration range of $0.5-4.0 \mu \mathrm{gHg} / 1$, the linear regression equation was $A=1.3628[\mathrm{Hg}]-0.1119$, and the correlation coefficient was 0.99 . The detection limit of mercury estimated from the peak height measurement was $0.15 \mu \mathrm{gHg} / 1(S /$ $N=3$ ). Figure 5 shows the atomic absorption responses of mercury $(4.0 \mu \mathrm{g} / 1)$ obtained by 10 injections. This result gives a relative standard deviation of $c a .1 \%$.

\section{Effect of sampling time on the mercury response}

Figure 6 shows some typical mercury signals at different pumping (sample injection) times of the sample solution in the determination of total mercury, where a standard solution of $4.0 \mu \mathrm{gHg} / 1$ and a blank solution were pumped alternately. The shape of the signal response changed from the transient signal to the steadystate signal when the sampling time was longer. Under these experimental conditions, the response reached a limiting value at 3 min pumping time (sample injection volume of about $600 \mu \mathrm{l}$ ) and the response time, i.e., the time required to obtain constant absorbance after the sample injection, was about $4 \mathrm{~min}$. This response time was judged to be fast enough for monitoring the change of the total mercury concentration in waste water.

\section{Continuous monitoring of mercury in waste water}

Continuous monitoring of total mercury content in waste water is important for environmental water-quality control. For such a purpose, the completely continuous flow monitoring is more desirable than the conventional discrete monitoring. On the basis of the studies described here, an automated prototype monitoring system was constructed. Waste water from the pond in Nagoya University campus was used as the sample. This. was supplied through a pipe for environmental monitoring by pumping to the laboratory in the Research

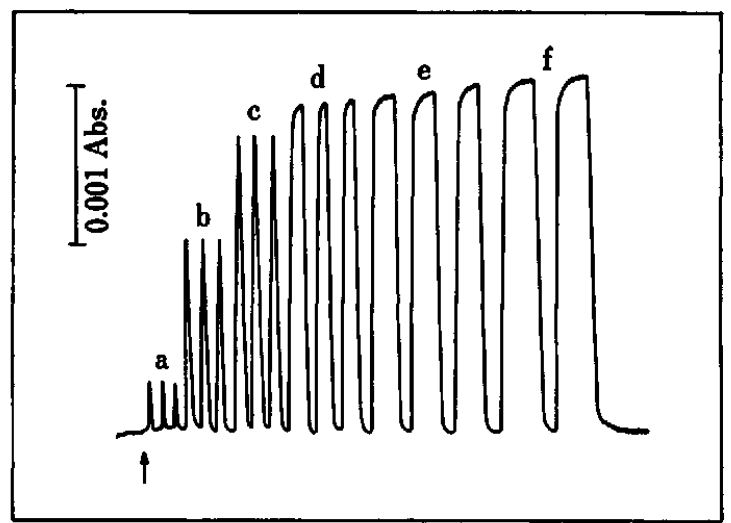

Fig. 6 Typical responses at different pumping times. Sample concentration, $4.0 \mu \mathrm{g} / \mathrm{l}$; pumping times: a. $15 \mathrm{~s}$, b. $45 \mathrm{~s}$, c. $1 \mathrm{~min}, \mathrm{~d} .2 \mathrm{~min}$, e. $6 \mathrm{~min}$, and f. $10 \mathrm{~min}$. 


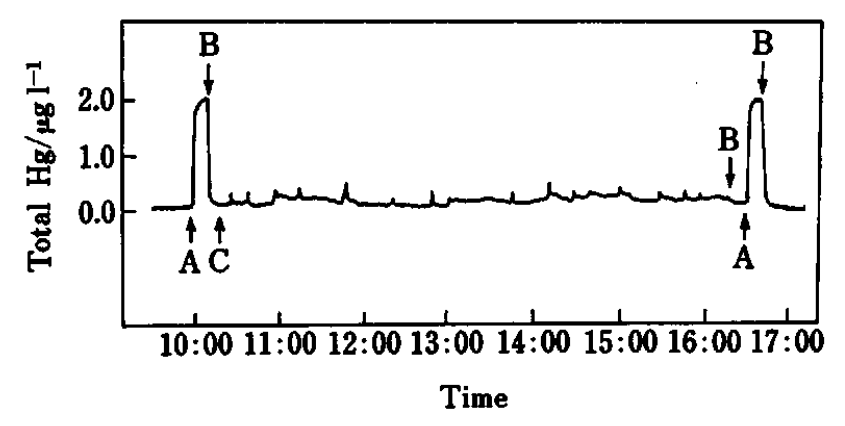

Fig. 7 Continuous microflow monitoring of total mercury in waste water from the Nagoya University pond. Solution pumped: A, standard solution $(2.0 \mu \mathrm{g} / \mathrm{l}) ; \mathrm{B}$, pure water; C, sample solution.

Center for Resource and Energy Conservation. The pipe was connected to the manifold $S$ in Fig. 1, and the sample was pumped continuously by using a peristaltic pump. The result is shown in Fig. 7. The stability of the mercury signal was checked by pumping a standard solution of $2.0 \mu \mathrm{gHg} / \mathrm{l}$ at the beginning and end of the measurements. As is seen in Fig. 7, the stability was good enough for at least $7 \mathrm{~h}$. It should be noted that a completely continuous flow monitoring of total mercury could be successfully performed by the present system. The mercury concentration in waste water was lower than $0.5 \mu \mathrm{g} / 1$.

In conclusion, the proposed method allows completely continuous flow and automated monitoring of total mercury in waste water for water-quality management. It should be emphasized that the amounts of the reagents required for the proposed system are much lower than the conventional automated one, because the flow rates of the reagents used are lower than $100 \mu \mathrm{l} / \mathrm{min}$.

One of the authors (EM) is grateful to the Japanese
Government for the award of a scholarship. The present research has been partly supported by the Grant-in-Aid for Scientific Research (No. 02202121) from the Ministry of Education, Science and Culture, Japan.

\section{References}

1. W. R. Hatch and W. L. Ott, Anal. Chem., 40, 2085 (1968).

2. M. Goto, E. Munaf and D. Ishii, Fresenius' Z. Anal. Chem., 332, 745 (1988).

3. E. Munaf, M. Goto and D. Ishii, Fresenius' Z. Anal. Chem., 334, 115 (1989).

4. E. Munaf, H. Haraguchi, D. Ishii, T. Takeuchi and $M$. Goto, Sci. Total Environ., 99, 205 (1990).

5. E. Munaf, $H$. Haraguchi, D. Ishii, T. Takeuchi and $M$. Goto, Anal. Chim. Acta, 235, 399 (1990).

6. E. Munaf, T. Takeuchi, M. Goto, H. Haraguchi and D. Ishii, Anal. Sci., 6, 313 (1990).

7. W. B. Robbins and J. A. Caruso, Anal. Chem., 51, 889A (1979).

8. R. G. Godden and D. R. Thomerson, Analyst [London], 105, 1137 (1980).

9. J. A. Holcombe and D. A. Bass, Anal. Chem., 60, 226R (1988).

10. D. R. Qiiu, C. Vandecasteele, K. Vermeiren and R. B. Dams, Spectrochim. Acta, 45B, 439 (1990).

11. M. Goto, T. Shibakawa, T. Arita and D. Ishii, Anal. Chim. Acta, 140, 179 (1982).

12. F. J. Fernandez, At. Absorption Newsl., 12, 93 (1973).

13. M. Fujita and E. Takabatake, Anal. Chem., 55, 454 (1983).

14. J. C. de Andrade, C. Pasquini, N. Baccan and J. C. Van Loon, Spectrochim. Acta, 38B, 1329 (1983).

15. R. C. Rooney, Analyst [London], 101, 678 (1976).

16. J. Toffaletti and J. Savory, Anal. Chem., 47, 2091 (1975).

17. Y. Yamamoto, T. Kumamaru and A. Shiraki, Fresenius' Z. Anal. Chem., 292, 273 (1978).

18. C. E. Oda and J. D. Ingle, Anal. Chem., 53, 2030 (1981).

19. L. Magos, Analyst [London], 96, 847 (1971).

(Received May 7, 1991) (Accepted June 19, 1991) 